\title{
Multivariate generalizations of the Foata-Schützenberger equidistribution
}

\author{
Florent Hivert ${ }^{1}$, Jean-Christophe Novelli ${ }^{2}$ and Jean-Yves Thibon ${ }^{2}$ \\ ${ }^{1}$ LITIS, Université de Rouen ; Avenue de l'université ; 76801 Saint Étienne du Rouvray, France \\ ${ }^{2}$ Institut Gaspard Monge, Université de Marne-la-Vallée ; 77454 Marne-la-Vallée cedex 2
}

A result of Foata and Schützenberger states that two statistics on permutations, the number of inversions and the inverse major index, have the same distribution on a descent class. We give a multivariate generalization of this property: the sorted vectors of the Lehmer code, of the inverse majcode, and of a new code (the inverse saillance code), have the same distribution on a descent class, and their common multivariate generating function is a flagged ribbon Schur function.

\section{Introduction}

The major index of a permutation, discovered by Major Percy Alexander MacMahon, and named after his military rank, is the sum of its descents

$$
\operatorname{maj}(\sigma)=\sum_{\sigma(i)>\sigma(i+1)} i
$$

The maximum value of maj over the set $\mathfrak{S}_{n}$ of permutations of size $n$ is $n(n-1) / 2$, the same as for the inversion number inv, and MacMahon proved [12] (actually, he proved a similar result for an arbitrary rearrangement class of words, but in this paper, we will only deal with permutations) that both statistics have the same distribution

$$
\sum_{\sigma \in \mathfrak{S}_{n}} q^{\operatorname{maj}(\sigma)} \sum_{\sigma \in \mathfrak{S}_{n}} q^{\operatorname{inv}(\sigma)}=[n]_{q} ! \prod_{i=1}^{n} \frac{1-q^{i}}{1-q} .
$$

More than fifty years later, Foata and Schützenberger [6] proved that equidistribution holds on a descent class:

$$
\sum_{\operatorname{Des}(\sigma)=D} q^{\operatorname{maj}\left(\sigma^{-1}\right)} \sum_{\operatorname{Des}(\sigma)=D} q^{\operatorname{inv}(\sigma)}
$$

where $\operatorname{Des}(\sigma)=\{i \mid \sigma(i)>\sigma(i+1)\}$ is the descent set of $\sigma$. The original proof (and, up to recently the only one, $c f$. [11], chapter 11) of this result was bijective. In this note, we obtain a multivariate refinement of (3): we prove that, up to order, the three integer vectors to be defined below, namely the inverse Lehmer code $\operatorname{Ic}\left(\sigma^{-1}\right)=\left(c_{1}, \ldots, c_{n}\right)$, the inverse major code $\operatorname{Mc}\left(\sigma^{-1}\right)=\left(m_{1}, \ldots, m_{n}\right)$, and a new code $\operatorname{Sc}\left(\sigma^{-1}\right)=\left(s_{1}, \ldots, s_{n}\right)$ have the same distribution on a descent class, that is, if $x_{0}, \ldots, x_{n-1}$ are independent indeterminates

$$
\sum_{\operatorname{Des}(\sigma)=D} \prod_{i} x_{c_{i}\left(\sigma^{-1}\right)} \sum_{\operatorname{Des}(\sigma)=D} \prod_{i} x_{m_{i}\left(\sigma^{-1}\right)} \sum_{\operatorname{Des}(\sigma)=D} \prod_{i} x_{s_{i}\left(\sigma^{-1}\right)} .
$$

Indeed, the codes are defined in such a way that

$$
\sum_{i=1}^{n} c_{i}\left(\sigma^{-1}\right)=\operatorname{inv}\left(\sigma^{-1}\right)=\operatorname{inv}(\sigma), \quad \sum_{i=1}^{n} m_{i}\left(\sigma^{-1}\right)=\operatorname{maj}\left(\sigma^{-1}\right)
$$

so that Equation (4) is a refinement of Equation (3). 
Acknowledgements.- This project has been partially supported by CNRS. The authors would also like to thank the contributors of the MuPAD project, and especially those of the combinat package, for providing the development environment for this research (see [8] for an introduction to MuPAD-Combinat).

\section{Notations}

\section{Alphabets and operations on words}

In all the paper, we deal with a totally ordered infinite alphabet $A$, represented either by $\{a, b, c, \ldots\}$ or by $\{1,2,3, \ldots\}$. The free associative algebra over $A$ is denoted by $\mathbb{K}\langle A\rangle$, where $\mathbb{K}$ is some field of characteristic zero. The evaluation $\mathrm{ev}(w)$ of a word $w$ of size $n$ over the alphabet $\{0, \ldots, n\}$ is the list of numbers of appearances $\operatorname{Card}\left\{i \mid w_{i}=a\right\}$ of all letters $a \in A$ in $w$. For example, the evaluation of 45143251812 is 032122001000 . We denote by $\operatorname{id}_{n}=12 \cdots n$ the identity permutation of size $n$.

The shuffle product $w_{1} \amalg w_{2}$ of two words $w_{1}$ and $w_{2}$ is recursively defined by $w_{1} \amalg \epsilon=w_{1}$ and $\epsilon \amalg w_{2}=$ $w_{2}$, where $\epsilon$ is the empty word, and

$$
a u \amalg b v=a(u \amalg b v)+b(a u \amalg v), \quad a, b \in A, \quad u, v \in A^{*} .
$$

For example,

$$
12 \amalg 43=1243+1423+1432+4123+4132+4312 .
$$

For a word $w=w_{1} \ldots w_{n}$ over the integers, and $k \in \mathbb{N}$, we denote by $w[k]$ the shifted word

$$
w[k]:=\left(w_{1}+k\right) \cdot\left(w_{2}+k\right) \cdots\left(w_{n}+k\right) .
$$

The shifted shuffle of two permutations $\alpha \in \mathfrak{S}_{k}$ and $\beta \in \mathfrak{S}_{l}$ is then defined by

$$
\alpha \uplus \beta:=\alpha \amalg(\beta[k]) .
$$

\section{Compositions}

A composition of an integer $n$ is a sequence of positive integers of sum $n$. The descent set $\operatorname{Des}(I)$ of a composition $I=\left(i_{1}, \ldots, i_{r}\right)$ is the set of partial sums $\left\{i_{1}, i_{1}+i_{2}, \ldots, i_{1}+\cdots+i_{r}\right\}$. Compositions are ordered by $I<J$ iff $\operatorname{Des}(I) \subset \operatorname{Des}(J)$. In this case, we say that $I$ is coarser than $J$.

The descent composition $I=C(\sigma)$ of a permutation $\sigma$ is the composition of $n$ whose descents are equal to the descents of $\sigma$, that is, the set of integers $j$ such that $\sigma(j)>\sigma(j+1)$.

If $I=\left(i_{1}, \ldots, i_{r}\right)$ is a composition of $n$, let $D_{\leq I}$ be the sum of all permutations whose descent composition is coarser than or equal to $I$. Then

$$
D_{\leq I}=\left(\operatorname{id}_{i_{1}} \uplus \operatorname{id}_{i_{2}} \uplus \cdots \cup \operatorname{id}_{i_{r}}\right)^{\vee}
$$

where $\vee$ is the linear involution sending each permutation to its inverse. The sum of all permutations whose descent composition is $I$ will be denoted by $D_{I}$.

Recall that the algebra of noncommutative symmetric functions Sym is defined as the free associative algebra on symbols $S_{n}$ so that a basis is given by the $S^{I}=S_{i_{1}} \cdots S_{i_{r}}$ for all compositions $I=$ $\left(i_{1}, \ldots, i_{r}\right)$ [7]. We will make use of this basis of $\mathbf{S y m}$ and of the ribbon basis $R_{I}$ defined by

$$
S^{I}:=\sum_{J \leq I} R_{J} .
$$

When $A$ is an ordered alphabet, $S_{n}(A)$ can be realized as the sum of all nondecreasing words in $A^{n}$. The commutative image of Sym is the algebra of symmetric functions. The $S_{n}$ are mapped to the usual complete homogeneous functions $h_{n}$, and the $R_{I}$ to the ribbon Schur functions $r_{I}$.

\section{Codes}

Let us say that a sequence $a=\left(a_{1}, \ldots, a_{n}\right)$ is sub-diagonal if $0 \leq a_{i} \leq n-i$. A code is a bijection between the symmetric group $\mathfrak{S}_{n}$ and the set of sub-diagonal sequences of length $n$. Among known codes, we will be interested in the Lehmer code and the major code.

Recall that the Lehmer code (or Lcode, for short) $\operatorname{Lc}(\sigma)$ of a permutation $\sigma \in \mathfrak{S}_{n}$ is the sequence $\left(c_{i}\right)_{1 \leq i \leq n}$, where

$$
c_{i}=\operatorname{Card}\left\{j>i \mid \sigma_{j}<\sigma_{i}\right\}
$$


For example, the code of the permutation 531962487 is 420520010.

The Lehmer code of the inverse permutation will be called the Inversion code (or invcode, for short). It is the sequence $\operatorname{Ic}(\sigma)=\left(a_{1}, \ldots, a_{n}\right)$, where $a_{i}$ is the number of values greater than $i$ to its left. In other words, the invcode, as the Lehmer code, splits the inversions of $\sigma$ into blocks.

Since the number of inversions of a permutation is the sum of the components of its code, one may look for an analogous vector having as sum the major index. The major code (or majcode, for short) solves this question. It is implicit in Carlitz [1] and explicitly stated by Rawlings in [13] (see also [14]). Recall that the major index maj of a permutation is the sum of the positions of its descents. Now, for $\sigma \in \mathfrak{S}_{n}$, denote by $\sigma^{(i)}$ the subword of $\sigma$ obtained by erasing the letters smaller than $i$, so that $\sigma=\sigma^{(1)}$. Then the majcode $\operatorname{Mc}(\sigma)$ of $\sigma$ is the sequence $\left(c_{i}\right)_{1 \leq i \leq n}$, where $c_{n}=0$ and

$$
c_{i}=\operatorname{maj}\left(\sigma^{(i)}\right)-\operatorname{maj}\left(\sigma^{(i+1)}\right),
$$

for all $1 \leq i \leq n-1$. For example, $\operatorname{Mc}(935721468)=501012010$.

Finally, the sorted vector $V^{\uparrow}$ of a vector $\left(v_{1}, \ldots, v_{n}\right)$ is its nondecreasing rearrangement.

\section{Cayley trees and codes}

\subsection{From differential equations to trees}

Cayley [2] introduced trees in order to solve the differential equation

$$
\frac{d x}{d t}(t)=V(x(t))
$$

where $V$ is a vector field, that is, a function from $\mathbb{R}^{d}$ to itself.

Formally, the special case $d=1$ gives the following values for the coefficients $x_{n}=d^{n} x / d t^{n}(0)$ of the Taylor expansion at $t=0$ of the solution:

$$
\begin{aligned}
x_{1} & =V_{0} \\
x_{2} & =V_{1} V_{0} \\
x_{3} & =V_{2} V_{0}^{2}+V_{1}^{2} V_{0} \\
x_{4} & =V_{3} V_{0}^{3}+4 V_{2} V_{1} V_{0}^{2}+V_{1}^{3} V_{0} \\
x_{5} & =V_{4} V_{0}^{4}+7 V_{3} V_{1} V_{0}^{3}+4 V_{2}^{2} V_{0}^{3}+11 V_{2} V_{1}^{2} V_{0}^{2}+V_{1}^{4} V_{0} \\
x_{6}=V_{5} V_{0}^{5}+11 V_{4} V_{1} V_{0}^{4}+15 V_{3} V_{2} V_{0}^{4}+32 V_{3} V_{1}^{2} V_{0}^{3}+34 V_{2}^{2} V_{1} V_{0}^{3} & +26 V_{2} V_{1}^{3} V_{0}^{2}+V_{1}^{5} V_{0}
\end{aligned}
$$

where $V_{n}$ is $\frac{d^{n} V}{d x^{n}}(x(0))$.

Assuming without loss of generality that $V_{0}=1$, we have in the one-dimensional case

$$
x_{n+1}=C_{n}\left(V_{1}, \ldots, V_{n}\right),
$$

where the polynomials $C_{n}$ reduce to the Eulerian polynomials

$$
C_{n}(q, \ldots, q)=A_{n}(q)
$$

when all the $V_{i}$ are equal to $q$.

The polynomials $C_{n}$ giving the Taylor coefficients of the unique solution of $\frac{d x}{d t}=V(x(t))$ with $x(0)=0$ and $V(0)=1$ should be compared to the exponential Bell polynomials

$$
B_{n}\left(x_{1}, \ldots, x_{n}\right)=\sum_{k} B_{n, k}\left(x_{1}, \ldots x_{n+1-k}\right)
$$

giving the Taylor coefficients of $y(t)=V(x(t))$

$$
y_{n}=\sum_{k=0}^{n} V_{k} B_{n, k}\left(x_{1}, \ldots, x_{n+1-k}\right) .
$$


Both calculations are related to the so-called Faà di Bruno Hopf algebra [5]. The $C_{n}$ are to the Eulerian polynomials what the $B_{n}$ are to the (one variable) Bell polynomials

$$
b_{n}(q)=B_{n}(q, \ldots, q) .
$$

It is immediate that the sum of the coefficients of the monomials $V^{\alpha}$ in $x_{n+1}$ is $n$ !, so that the exponent vector $\alpha$ should be interpretable as a multivariate statistic on the symmetric group $\mathfrak{S}_{n}$. Alain Lascoux observed that this statistic seems to coincide with the sorted evaluation $\operatorname{ev}(\operatorname{Lc}(\sigma))^{\uparrow}$ of the Lehmer code, which is fundamental in the theory of Schubert and Grothendieck polynomials [9]. The codes of the permutations of $\mathfrak{S}_{3}$ are

$$
000,010,100,110,200,210
$$

whose evaluations are

$$
\text { 3000, 2100, 2100, 1200, 2010, 1110, }
$$

giving back the exponents of $x_{4}$. More generally, one has

$$
x_{n}=\sum_{\sigma \in \mathfrak{S}_{n-1}} V^{\mathrm{Ic}(\sigma)} .
$$

We shall see that a better way to understand this formula is to rely upon another statistic on permutations coming up more naturally than the Lehmer code from differential equations, namely the saillance code. It comes from decreasing tree structures on permutations appearing in the $d$-dimensional case of Equation 14 . When $d>1$, the derivatives $V_{i}$ have to be replaced by the differentials $D^{k} V$ defined by

$$
\left[D^{k} V\left(U_{1}, \ldots U_{k}\right)\right]_{i}: \sum_{j_{1} \ldots j_{k}=1}^{d} \frac{\partial^{k}[V]_{i}}{\partial x_{j_{1}} \ldots \partial x_{j_{k}}}\left[U_{1}\right]_{j_{1}} \ldots\left[U_{k}\right]_{j_{k}}
$$

where $U_{j}$ is any vector field and $\left[U_{j}\right]_{i}$ denotes the $i$-th coordinate of $U_{j}$. If the $U_{i}$ are evaluated at $x(t)$,

$$
\frac{d\left(D^{k} V\left(U_{1}, \ldots, U_{k}\right)\right)}{d t}=D^{k+1} V\left(U_{1}, \ldots, U_{k}, V\right)+\sum_{i=1}^{k} D^{k} V\left(U_{1}, \ldots, \frac{d U_{i}}{d t}, \ldots, U_{k}\right)
$$

The calculation of the Taylor coefficients $x_{n+1}=d^{n} / d t^{n} V(x(t))$ at $t=0$ gives rise to nested derivatives, which can be conveniently represented by (unlabelled rooted) trees (sequence A000081 in [15]).

Define $D_{T} V$ recursively by: if the root of $T$ has $k$ subtrees $T_{1}, \ldots, T_{k}$,

$$
D_{T} V:=D^{k} V\left(D_{T_{1}} V, \ldots, D_{T_{k}} V\right),
$$

with the convention $D^{0} V=V$.

For example,

$$
D^{2} V\left(V, D^{3} V\left(V, D^{2} V(V, V), V\right)\right)=\overbrace{V}^{D^{2} V}
$$

With this notation, the derivation rule becomes

$$
\frac{d\left(D_{T} V\right)}{d t}=\sum_{T^{\prime}} D_{T^{\prime}} V
$$

where $T^{\prime}$ runs over the set of trees obtained from $T$ by adding a leaf to a node of $T$. For example, writing only the trees in the previous equation, one has

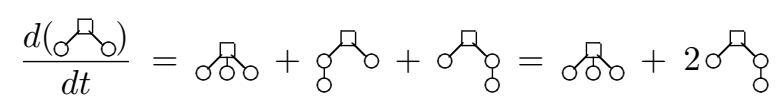

We have thus, for each integer $n$, an expression

$$
x_{n}=\sum_{|T|=n} \alpha_{T} D_{T} V,
$$


where the $\alpha_{T}$ are positive integers, sometimes known as the Connes-Moscovici coefficients [4, 3].

The first values are

$$
\begin{aligned}
& x_{1}=\bullet \\
& x_{2}=\text { 뭉 } \\
& x_{3}=\text { 웅 只 }
\end{aligned}
$$

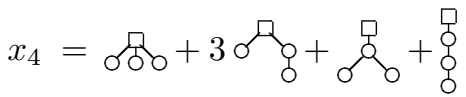

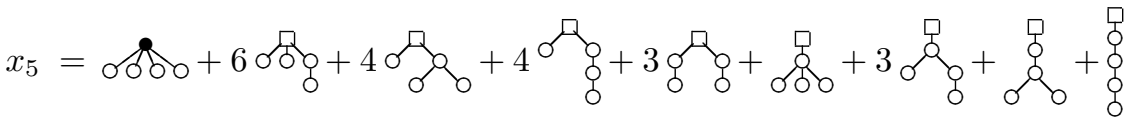

\subsection{From trees to permutations and statistics}

It is easy to see that the coefficient $\alpha_{T}$ is equal to the number of increasing trees of shape $T$, that is, the set of trees obtained by labelling the nodes of $T$ by the integers from 1 to $n$ so that the label of each node is greater than the label of its parent. Recall that increasing trees have a canonical form that consists in ordering the children of each node so that they are increasing from left to right. For example, written in canonical form, here are the five increasing trees of a given shape.<smiles>[2H]I=[V][3H]</smiles><smiles>[O-][Tl](I)[Hg]</smiles>

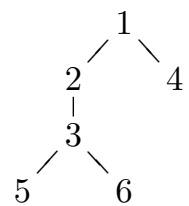<smiles></smiles>

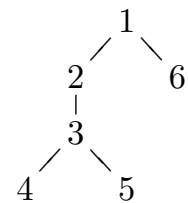

Recall that the total number of increasing trees of size $n$ is $(n-1)$ !. There exist many different bijections with permutations of size $n-1$. We shall make use of the following one. Start from an increasing tree of size $n$, replace each label $l$ in $T$ by $n+1-l$, then reorder the (now decreasing) tree in canonical form and read it in prefix order, forgetting the root. For example, the permutations corresponding to the increasing trees of (41) are

$$
\text { 43125, } \quad \text { 45312, } \quad 35412, \quad 25413, \quad 15423 .
$$

The polynomials 21 (for $d=1$ ) are obtained by replacing each tree $T$ by the monomial

$$
\prod_{o \in \operatorname{nodes}(T)} V_{\operatorname{arity}(o)} .
$$

The resulting statistic on trees can be interpreted as the evaluation of a code:

Definition 3.1 The saillance code (or scode, for short) $\operatorname{Sc}(T)$ of a tree $T$ of size $n$ as the sequence of labels of the parents (minus one) of $n, n-1, \ldots, 2$.

For example, the scode of the trees of 41 are

$$
33200, \quad 22010, \quad 20210, \quad 02210 .
$$

\section{Properties of the scode}

The scode can be directly defined on permutations as follows: the scode of a permutation $\sigma \in \mathfrak{S}_{n}$ is the sequence $a=\left(a_{1}, \ldots, a_{n}\right)$, where $a_{i}$ is the number of letters of $\sigma$ greater than or equal to the rightmost letter to the left of $i$ and greater than $i$.

For example, one can check that the scodes of the permutations of (42) are the sequences of (44).

Proposition 4.1 The scode is a code.

Proof-Since $a_{i}$ counts a number of letters in $\sigma$ all greater than $i$, the sequence $a$ is sub-diagonal. Moreover, thanks to the bijection between permutations and increasing trees, and to the fact that the scode is obviously injective from trees, the scode is a bijection.

The algorithm giving back the permutation from its scode $a=\left(a_{1}, \ldots, a_{n}\right)$ is as follows: put $n$ and then insert letters $n-1$ to 1 such that $i$ is inserted immediately after letter $n+1-a_{i}$ (and first if $a_{i}=0$ ). 
Proposition 4.2 Interpreting a sub-diagonal sequence as the scode of a permutation, the number of descents of this permutation is given by the number of distinct non-zero values in its scode.

Proof - The number of descents of a permutation is equal to the number of internal nodes except for the root of the corresponding increasing tree, which is clearly equal to the number of distinct non-zero values of its scode.

It is known that the generating function of the Lehmer code (up to order) over a class $D_{\leq I}$ admits a closed expression, as a product of complete symmetric functions over a flag of alphabets. This property is also true for the scode. Let us first introduce for all $n \geq 0$, the alphabet $X_{n}=\left\{x_{0}, \ldots, x_{n}\right\}$ where the $x_{i}$ are commuting indeterminates. With a given sub-diagonal sequence $c$, we associate the monomial

$$
x_{c}=x_{c_{1}} x_{c_{2}} \cdots x_{c_{n}} \in X_{n-1} \times X_{n-2} \times \cdots \times X_{0} .
$$

Theorem 4.3 Let $I=\left(i_{1}, \ldots, i_{r}\right)$ be a composition of $n$. The sum of the scodes of the inverses of the elements of $D_{\leq I}$ are given by the generating function

$$
\begin{aligned}
& F_{S}(I):=\sum_{\sigma \in D_{\leq I}} x_{\mathrm{Sc}\left(\sigma^{-1}\right)} \sum_{\sigma \in \operatorname{id}_{i_{1}} \mathbb{\cdots} \in \mathrm{id}_{i_{r}}} x_{\mathrm{Sc}(\sigma)} \\
& =h_{i_{1}}\left(X_{i_{2}+\cdots+i_{r}}\right) h_{i_{2}}\left(X_{i_{3}+\cdots+i_{r}}\right) \cdots h_{i_{r-1}}\left(X_{i_{r}}\right) h_{i_{r}}\left(X_{0}\right) \text {. }
\end{aligned}
$$

The right-hand side will be denoted by $h^{I}\left(\mathbf{X}_{I}\right)$, where $\mathbf{X}_{I}$ denotes the flag of alphabets

$$
\left(X_{n-i_{1}}, X_{n-i_{1}-i_{2}}, \ldots, X_{n-n}\right) \text {. }
$$

The proof relies on the following lemmas.

Let $\beta \in \mathfrak{S}_{n}$. For $0 \leq i \leq n$, denote by $1 \cup_{i} \beta$ the term of the shifted shuffle $1 \cup \beta$ in which 1 occurs at the $(i+1)$-st position, e.g., $1 \cup_{0} 21=132 ; \quad 1 \cup_{1} 21=312 ; \quad 1 \cup_{2} 21=321$.

Lemma 4.4 Let $\beta \in \mathfrak{S}_{n}$. Then

$$
x_{\mathrm{Sc}\left(1 \uplus_{i} \beta\right)}=x_{\tau_{S}(\beta)(i)} x_{\mathrm{Sc}(\beta)},
$$

where $\tau_{S}(\beta)$ is the permutation of $\{0, \ldots, n\}$ defined by

$$
\tau_{S}(\beta)(0)=0, \quad \text { and } \quad \tau_{S}(\beta)(i)=n+1-\beta(i) .
$$

For example,

$$
\begin{aligned}
\beta & =941625738 \\
\tau_{S}(\beta) & =0169485372
\end{aligned}
$$

Proof-Let $\beta^{\prime}=1 \uplus_{i} \beta$. It is obvious that $\operatorname{Sc}_{i+1}\left(\beta^{\prime}\right)=\operatorname{Sc}_{i}(\beta)$ for $i \in[1, n]$. So $x_{\mathrm{Sc}\left(\beta^{\prime}\right)} / x_{\mathrm{Sc}(\beta)}=x_{\mathrm{Sc}_{1}\left(\beta^{\prime}\right)}$. The rightmost value to the left of 1 and greater than 1 is its neighbour to the left. And the number of values greater than or equal to this last value is its complement to $n+1$, so that it corresponds to the definition of $\tau$.

Lemma 4.5 Let $\beta \in \mathfrak{S}_{n}$, let $k$ be an element of $[0, n]$ and let $\beta^{\prime}=1 \cup_{k} \beta$. Then

$$
\tau_{S}\left(\beta^{\prime}\right)(i)=\tau_{S}(\beta)(i)
$$

for $i \in[0, k]$.

For example, given $\beta=72451836$, we have $\tau_{S}(\beta)=027548163$. The case $k=3$ gives $\beta^{\prime}=835162947$ and $\tau_{S}\left(\beta^{\prime}\right)=0275948163$. The case $k=7$ gives $\beta^{\prime}=835629417$ and $\tau_{S}\left(\beta^{\prime}\right)=0275481693$.

Lemma 4.6 Let $\beta \in \mathfrak{S}_{n}$. Then the scodes of the elements in $i d_{k} \uplus \beta$ are

$$
\left(\tau_{S}(\beta)\left(i_{1}\right), \tau_{S}(\beta)\left(i_{2}\right), \ldots, \tau_{S}(\beta)\left(i_{k}\right), \operatorname{Sc}(\beta)\right)
$$

where $\left(i_{j}\right)$ runs over nondecreasing sequences in $[0, n], 0 \leq i_{1} \leq i_{2} \leq \ldots \leq i_{k} \leq n$.

In particular, we have

$$
\sum_{\sigma \in \mathrm{id}_{k} \uplus \beta} x_{\mathrm{Sc}(\sigma)}=h_{k}\left(X_{n}\right) x_{\mathrm{Sc}(\beta)} .
$$


Proof - It is sufficient to prove the result for $k=2$. The computation of $12 \cup \beta$ can be decomposed as the shifted shuffle of 1 with $\beta$ followed by the shifted shuffle of 1 with the new elements where 1 cannot go to the right of 2 . Since the set of values $\tau_{S}\left(\beta^{\prime}\right)(i)$ for all $i$ in $[0, k]$ where $k$ is the position of 1 in $\beta^{\prime}$ is equal to the set of values $\tau_{S}(\beta)(i)$ for all $i$ in $[0, k]$, we are done for the first part of the lemma.

Then, since $\tau$ is a permutation of $[0, n]$, the commutative image of the sum of all words $a_{\tau_{\beta}\left(i_{1}\right)} \ldots a_{\tau_{\beta}\left(i_{k}\right)}$ where $\left(i_{j}\right)$ are the nondecreasing sequences, is $h_{k}\left(X_{n}\right)$.

A slightly more general result can be derived from the previous considerations: if one considers a noncommutative ordered alphabet $A_{n}\left\{a_{0}<\ldots<a_{n}\right\}$ instead of $X_{n}$, so that one associates with a subdiagonal word $c$ the word $a_{c}=a_{c_{1}} a_{c_{2}} \cdots a_{c_{n}}$, the noncommutative series generalizing Equation (53) reads

$$
\sum_{\sigma \in \operatorname{id}_{k} \uplus \beta} a_{\operatorname{Sc}(\sigma)}=S_{k}\left(A_{n}^{\prime}\right) a_{\operatorname{Sc}(\beta)},
$$

where $A_{n}^{\prime}$ is the ordered alphabet on $\left\{a_{0}, \ldots, a_{n}\right\}$ where $a_{i}<a_{j}$ if $i$ is to the left of $j$ in $\tau_{S}(\beta)$. This property follows directly from Equation (51).

\section{Noncommutative and commutative generating function for codes}

We have already mentioned that the evaluations of the scodes and of the invcodes are the same over the symmetric group, which is obvious since both are codes and hence run over the set of sub-diagonal words. This proves the observation of Lascoux. Actually, the scode and the invcode have much more in common. The key result is that the sorted vectors $\operatorname{Sc}(\sigma)^{\uparrow}$ and $\operatorname{Ic}(\sigma)^{\uparrow}$ have the same distribution on inverse descent classes, a property also shared by the majcode (see Section 6). Equation (46) of Theorem 4.3 gives the closed expression of the generating function of these statistics.

Theorem 5.1 Let $I=\left(i_{1}, \ldots, i_{r}\right)$ be a composition of $n$. The sum of the invcodes of the inverses of the elements of $D_{\leq I}$ is given by the noncommutative generating function

$$
\begin{aligned}
F_{S}(I):=\sum_{\sigma \in D \leq I} a_{\operatorname{Ic}\left(\sigma^{-1}\right)} & \sum_{\sigma \in \operatorname{id}_{i_{1}} \amalg \cdots \uplus \mathrm{id}_{i_{r}}} a_{\mathrm{Ic}(\sigma)} \\
& =S_{i_{1}}\left(A_{i_{2}+\cdots+i_{r}}\right) S_{i_{2}}\left(A_{i_{3}+\cdots+i_{r}}\right) \cdots S_{i_{r-1}}\left(A_{i_{r}}\right) S_{i_{r}}\left(A_{0}\right) .
\end{aligned}
$$

This right-hand side will be denoted by $S^{I}\left(\mathbf{A}_{I}\right)$, where $\mathbf{A}_{I}$ denotes the flag of alphabets

$$
\left(A_{n-i_{1}}, A_{n-i_{1}-i_{2}}, \ldots, A_{n-n}\right) .
$$

Proof - The proof proceeds by induction on the number of parts of $I$. If $I$ has one part, the only permutation is the identity and the statement is obvious. Assuming the result for the composition $\left(i_{2}, \ldots, i_{r}\right)$, let us prove it for $I$. First, let $\sigma$ be an element of $\operatorname{id}_{i_{2}} \cup \ldots \cup \operatorname{id}_{i_{r}}$ and let $\gamma$ be any element in $\operatorname{id}_{i_{1}} \cup \sigma$. Then, $\mathrm{Ic}_{i_{1}+k}(\gamma)=\mathrm{Ic}_{k}(\sigma)$ for all $k$. Moreover, the sequence $\mathrm{Ic}_{k}(\gamma)$ for $k \in\left[1, i_{1}\right]$ is nondecreasing, since $1, \ldots, i_{1}$ are in this order in $\gamma$, and it is bounded by the number of letters of $\sigma$, that is, $i_{2}+\cdots+i_{r}$.

Since the invcode is a bijection, no two words $\gamma$ can have the same code, hence the same first $i_{1}$ values, since the other ones are identical. Finally, the number of elements in $\operatorname{id}_{i_{1}} \uplus \sigma$ is equal to the number of nondecreasing sequences of size $k$ in $\left[0, i_{2}+\cdots+i_{r}\right]$ (a binomial coefficient), so that all sequences appear, and the sum of the invcodes of all elements in $\operatorname{id}_{i_{1}} \uplus \sigma$ is $S_{i_{1}}\left(A_{i_{2}+\cdots+i_{r}}\right) a_{\operatorname{Ic}(\sigma)}$.

Corollary 5.2 The invcodes of the permutations in an inverse descent class are given by

$$
\sum_{\sigma \in D_{I}} a_{\mathrm{Ic}\left(\sigma^{-1}\right)}=\sum_{J \leq I}(-1)^{l(I)-l(J)} S^{J}\left(\mathbf{A}_{J}\right)=: R_{I}\left(\mathbf{A}_{I}\right) .
$$

Taking the commutative image $\left(a_{i} \rightarrow x_{i}\right)$, we recover the following expression (see [11], chap. 11):

Corollary 5.3 The commutative generating series for the codes on a descent class is given by the following determinant (a flagged ribbon Schur function)

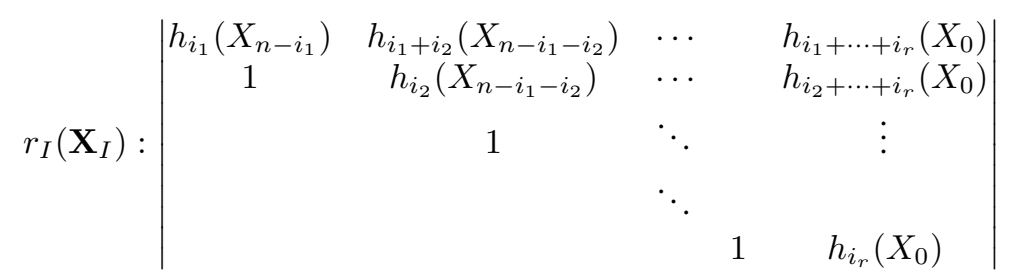


It is interesting to observe that this flagged Schur function is in fact a Schubert polynomial [9, 10]. For example, with $I=(5,1,2)$, one gets the following determinant:

$$
r_{512}\left(X_{3}, X_{2}, X_{0}\right)\left|\begin{array}{ccc}
h_{5}\left(X_{3}\right) & h_{6}\left(X_{2}\right) & h_{8}\left(X_{0}\right) \\
1 & h_{1}\left(X_{2}\right) & h_{3}\left(X_{0}\right) \\
0 & 1 & h_{2}\left(X_{0}\right)
\end{array}\right|=Y_{20150000}
$$

Corollary 5.4 The commutative generating series of $\operatorname{Sc}\left(\sigma^{-1}\right)$ on a descent class coincides with that of $\operatorname{Ic}\left(\sigma^{-1}\right)$ :

$$
\sum_{\sigma \in D_{I}} x_{\operatorname{Sc}\left(\sigma^{-1}\right)} \sum_{\sigma \in D_{I}} x_{\mathrm{Ic}\left(\sigma^{-1}\right)}=r_{I}\left(\mathbf{X}_{I}\right) .
$$

In other words, the sorted vectors $\operatorname{Ic}\left(\sigma^{-1}\right)^{\uparrow}$ and $\operatorname{Sc}\left(\sigma^{-1}\right)^{\uparrow}$ have the same distribution on a descent class.

For example, 61, 62, 63) present the 19 permutations with descent composition $(2,1,1,2)$ and their the invcodes and inverse scodes. Both statistics give the sequences of (64) when sorted.

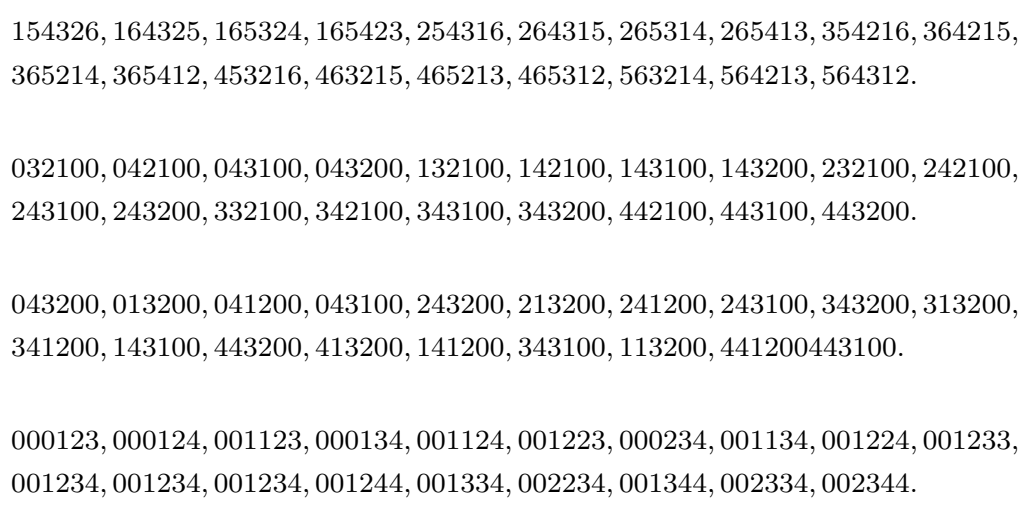

Note that one can reinforce the parallel between the scode and the invcode by observing that the sequence of lemmas used in the scode case could be directly translated in the invcode one, the only modification being the definition of $\tau_{S}$, which would be here

$$
\tau_{I}(\beta)=(0,1, \ldots, n)
$$

The fact that $\tau_{I}$ does not depend on $\beta$ and satisfies the same property as in Equation 51 is a satisfactory way to explain why the invcode has a noncommutative formula over the shuffle classes whereas $\tau_{S}$ has not. We shall see in the next Section that Equation (51) does not hold for the $\tau_{M}$, the permutation corresponding to the majcode and that this property explains why there is no noncommutative formula for this code.

\section{Generating function for majcodes}

Theorem 6.1 The commutative generating function of $\mathrm{Mc}\left(\sigma^{-1}\right)$ on a descent class coincide with that of $\operatorname{Ic}\left(\sigma^{-1}\right)$ :

$$
\sum_{\sigma \in D_{I}} x_{\mathrm{Mc}\left(\sigma^{-1}\right)}=\sum_{\sigma \in D_{I}} x_{\mathrm{Ic}\left(\sigma^{-1}\right)}=r_{I}\left(X_{I}\right)
$$

In other words, the sorted vectors $\operatorname{Mc}\left(\sigma^{-1}\right)^{\uparrow}$ and $\operatorname{Ic}\left(\sigma^{-1}\right)^{\uparrow}$ have the same distribution on a descent class.

For example, 67 presents the majcodes of the 19 permutations of (61). This statistic also gives the sequences of 64 when sorted.

$$
\begin{aligned}
& 332100,342100,343100,343200,232100,242100,243100,443200,132100,142100, \\
& 443100,243200,032100,442100,143100,143200,042100,043100,043200 .
\end{aligned}
$$

By inclusion-exclusion, the statement of the theorem is equivalent to

$$
\sum_{\sigma \in \operatorname{id}_{i_{1}} \uplus \cdots \uplus \mathrm{id}_{i_{r}}} x_{\mathrm{Mc}(\sigma)} \sum_{\sigma \in \mathrm{id}_{i_{1}} \uplus \cdots \uplus \mathrm{id}_{i_{r}}} x_{\mathrm{Ic}(\sigma)}
$$

for all compositions $I=\left(i_{1}, \ldots, i_{r}\right)$. This result is a consequence of the next four lemmas. 
Lemma 6.2 Let $\beta \in \mathfrak{S}_{n}$. Then

$$
x_{\mathrm{Mc}\left(1 \uplus_{i} \beta\right)}=x_{\tau_{M}(\beta)(i)} x_{\mathrm{Mc}(\beta)},
$$

where $\tau_{M}(\beta)$ is the permutation of $\{0, \ldots, n\}$ defined by

$$
\begin{aligned}
\tau_{M}(\beta)(i)=\operatorname{des}(\beta)-j & \text { if } i \text { is the } j \text {-th descent of } \beta, \\
\tau_{M}(\beta)(i)=\operatorname{des}(\beta)+j-1 & \text { if } i \text { is the } j \text {-th rise of } \beta,
\end{aligned}
$$

where the first position is considered as a rise, and des denotes the number of descents.

For example,

$$
\begin{aligned}
\beta & =941625738 \\
\tau_{M}(\beta) & =4325167089
\end{aligned}
$$

Note that $\tau_{M}(\beta)$ only depends on the descent composition $J$ of $\beta$ so that we can also denote it by $\tau_{M}(J)$. Proof-Let $\beta^{\prime}=1 \cup_{i} \beta$. It is obvious that $m_{i+1}\left(\beta^{\prime}\right)=m_{i}(\beta)$ for $i \in[1, n]$. So $x_{\operatorname{Mc}\left(\beta^{\prime}\right)} / x_{\operatorname{Mc}(\beta)}=x_{m_{1}\left(\beta^{\prime}\right)}$. Now, if $i$ is a descent of $\beta$, the insertion of 1 shifts by one position all the descents to its right. So, in this case, $x_{m_{1}\left(\beta^{\prime}\right)}=x_{\tau_{M}(\beta)(i)}$. Otherwise, if $i=0$, it shifts all descents of $\beta$ by one, so the same formula holds. Finally, between a rise $i$ and the next rise $j$ in $\beta$, we have $x_{m_{1}\left(1 \uplus_{j} \beta\right)}-x_{m_{1}\left(1 \uplus_{i} \beta\right)}=1$ since the first one creates a descent at position $j$ instead of position $i$ and shifts $j-i-1$ descents $(j$ is the next rise after $i$ ) less than $1 \cup_{i} \beta$. So the formula holds for all $i$.

Lemma 6.3 Let $\beta \in \mathfrak{S}_{n}$ and let $k$ be an element of $[0, n]$. The set $\left\{\tau_{M}(\beta)(i)\right\}_{i \in[0, k]}$ is the interval $[d, d+k]$, where $d$ is the number of descents of $\beta$ greater than $k$.

Proof - By construction of $\tau_{M}(\beta)$, it is obvious that this set is an interval of $k+1$ elements. Since its smallest element is the number of descents of $\beta$ greater than $k$, obtained either at the last descent of $\beta$ smaller than or equal to $k$ or at the first rise of $\beta$, we are done.

From this, we get immediately:

Lemma 6.4 Let $\beta \in \mathfrak{S}_{n}$, let $k$ be an element of $[0, n]$ and let $\beta^{\prime}=1 \mathbb{U}_{k} \beta$. Then,

$$
\left\{\tau_{M}\left(\beta^{\prime}\right)(i) \mid i \in[0, k]\right\}=\left\{\tau_{M}(\beta)(i) \mid i \in[0, k]\right\} .
$$

For example, given $\beta=72451836$, we have $\tau_{M}(\beta)=324516078$. The case $k=5$ gives $\beta^{\prime}=835621947$ and $\tau_{M}\left(\beta^{\prime}\right)=4356217089$. The case $k=6$ gives $\beta^{\prime}=835629147$ and $\tau_{M}\left(\beta^{\prime}\right)=3245160789$.

Lemma 6.5 Let $\beta \in \mathfrak{S}_{n}$ and $k$ be an integer. The set of the sorted $k$ first components of the majcodes of the elements in $i d_{k} \cup \beta$ is the set of all sequences $\left(0 \leq i_{1} \leq i_{2} \leq \cdots \leq i_{k} \leq n\right)$. In particular, we have

$$
\sum_{\sigma \in \operatorname{id}_{k} \uplus \beta} x_{\operatorname{Mc}(\sigma)}=h_{k}\left(X_{n}\right) x_{\mathrm{Mc}(\beta)} .
$$

Proof - The first part is proved by induction on $k$. For $k=1$, the result is obvious. Assume that it is true for $k-1$. We prove that for all nondecreasing sequences $\left(0 \leq i_{1} \leq i_{2} \leq \cdots \leq i_{k} \leq n\right)$, exactly one of its permutations can be obtained as the $k$ first components of the majcode of an element in $i d_{k} \cup \beta$. Consider a permutation satisfying this property. Then the first component of its majcode is necessarily the value $i_{j}$ that is in the rightmost position in $\tau_{M}(\beta)$ since otherwise this value could not be anywhere else in the majcode of this permutation thanks to Equation (70). We can then conclude by induction, since there are as many nondecreasing sequences of size $k$ from 0 to $n$ as elements in the shuffle $i d_{k} \cup \beta$. Then, since $\tau_{G}$ is a permutation of $[0, n]$, the commutative image of the sum of the $k$ first components of the majcodes is $h_{k}\left(X_{n}\right)$.

In the case of the majcode, no noncommutative formula holds, even for $i d_{k} \cup \beta$. Indeed, there is no fixed order on the set $\{0, \ldots, n\}$ as in the case of the scode associated with $\beta$. For example, 1423 belongs to $123 \cup 1$ and its scode is 1010 so that $1<0$ and $0<1$, which is impossible. 


\section{Codes and Euler-Mahonian statistics}

Let us say that a general code Gc is compatible with the shuffle if the codes of $1 \cup \beta$ are of the form $(i, \operatorname{Gc}(\beta))$. Since the code is a bijection, one can then define $\tau(\beta)$ as the permutation of $\{0, \ldots, n\}$ obtained by sending $i$ to the first value of the code of $1 \cup_{i} \beta$. For example, the scode, the invcode, and the majcode are compatible with the shuffle, the corresponding permutations $\tau(\beta)$ being respectively as defined in Equations (49, , 65, , 70, We then say that a general code Gc compatible with the shuffle is an acceptable code if, for all permutations $\beta$ and all integers $k$,

$$
\left\{\tau_{G}\left(\beta^{\prime}\right)(i) \mid i \in[0, k]\right\}=\left\{\tau_{G}(\beta)(i) \mid i \in[0, k]\right\} .
$$

where $\beta^{\prime}=1 \uplus_{k} \beta$. For example, the scode, the invcode, and the majcode are acceptable codes, as it is trivial for the invcode and already done in Lemma 4.5 for the scode and in Lemma 6.4 for the majcode.

The following Lemma is proved exactly as Lemma 6.5 , by induction on $k$.

Lemma 7.1 Let us consider an acceptable code Gc.

Let $\beta \in \mathfrak{S}_{n}$ and $k$ be an integer. Then consider the set of the sorted $k$ first components of the Gcodes of the elements in $i d_{k} \uplus \beta$. This set is exactly the set of all sequences

$$
\left(0 \leq i_{1} \leq i_{2} \leq \cdots \leq i_{k} \leq n\right)
$$

In particular, we have

$$
\sum_{\sigma \in \operatorname{id}_{k} \uplus \beta} x_{\mathrm{Gc}(\sigma)}=h_{k}\left(X_{n}\right) x_{\mathrm{Gc}(\beta)} .
$$

This implies the following theorem, which contains Theorems 4.3 and 5.4 , and Corollary 6.1 as particular cases.

Theorem 7.2 Let us consider an acceptable code Gc. Then the commutative generating series of $\operatorname{Gc}\left(\sigma^{-1}\right)$ on a descent class coincides with that of $\operatorname{Ic}\left(\sigma^{-1}\right)$ :

$$
\sum_{\sigma \in D_{I}} x_{\mathrm{Gc}\left(\sigma^{-1}\right)}=\sum_{\sigma \in D_{I}} x_{\mathrm{Ic}\left(\sigma^{-1}\right)}=r_{I}\left(X_{I}\right) .
$$

In other words, the sorted vectors $\operatorname{Gc}\left(\sigma^{-1}\right)^{\uparrow}$ and $\operatorname{Ic}\left(\sigma^{-1}\right)^{\uparrow}$ have the same distribution on a descent class.

Corollary 7.3 Let Gc be an acceptable code. Then the bi-statistic

$$
\left(\sum_{i \in \operatorname{Gc}\left(\sigma^{-1}\right)} i, \quad \operatorname{des}(\sigma)\right)
$$

is Euler-Mahonian.

\section{An equivalence related to sorted codes}

The previous sections showed the importance of the sorted codes. We present here a simple construction relating all permutations having a given sorted Lcode. Let us say that two words $u$ and $v$ are L-adjacent if there exists four words $w_{1}, w_{2}, w_{3}, w_{4}$ and three letters $a<b<c$ such that

$$
\begin{aligned}
& u=w_{1} a w_{2} c w_{3} b w_{4}, \\
& v=w_{1} b w_{2} a w_{3} c w_{4},
\end{aligned}
$$

where all letters of $w_{2}$ are greater than $b$, and all letters of $w_{3}$ and $w_{4}$ are either smaller than $b$ or greater than $c$. The L-equivalence is the transitive closure of the relation of L-adjacency. That is, two words $u, v$ are L-equivalent if there exists a chain of words $u=u_{1}, u_{2}, \ldots, u_{k}=v$, such that $u_{i}$ and $u_{i+1}$ are L-adjacent for all $i$. In this case, we write $u \sim v$. For example, the L class of $w=31452$ is the set $\{13542,14352$, $21543,23514,24153,24315,31452,32154,32415\}$. This is not a congruence on $A^{*}$.

Lemma 8.1 If $u$ and $v$ are L-adjacent, the sorted Lcode of $u$ and $v$ are equal. 
Proof - It is easy to check that the parts of the code corresponding to the subwords $w_{i}$ are the same in the code of $u$ and in the code of $v$. Moreover, the part of the code corresponding to $a$ (resp. $b, c$ ) in $u$ is the same as the part of the code corresponding to $a$ (resp. $c, b$ ) in $v$.

For example 738694152 and 758634192 are L-adjacent $(a=3, b=5, c=9)$ and their Lcodes are $\operatorname{Lc}(u)=625442010$ and $\operatorname{Lc}(v)=645422010$.

Lemma 8.2 Let $w$ be a word avoiding the pattern 132. Then there exists $w^{\prime}$ greater than $w$ in the lexicographic order such that $w$ and $w^{\prime}$ are L-adjacent.

Proof - Let $w$ be a word containing the pattern 132. Then there exists an occurrence of the pattern $a c b$ such that the difference $c-b$ is minimal. Then take $a$ as the rightmost element smaller than $b$ to the left of $c$. This element is L-adjacent to the element obtained by changing $a$ into $b, c$ into $a$ and $b$ into $c$. Indeed, by construction, all the words $w_{2}, w_{3}$, and $w_{4}$ satisfy the hypotheses of L-adjacency.

Proposition 8.3 Two words $u$ and $v$ are L-equivalent iff $u$ and $v$ have same sorted Lcode.

Proof - The number of sorted Lcodes is a Catalan number, also counting the permutations avoiding 132. By Lemma 8.1, the number of $\mathrm{L}$ classes is greater than or equal to the Catalan number. By Lemma 8.2. the number of $L$ classes is smaller than or equal to the same Catalan number. So the number of $L$ classes is given by the same Catalan number and the classes are the same as the classes of words by sorted Lcode.

Hence, each L class contains exactly one permutation avoiding 132 (its greatest element). In the same way, one can prove that each L class contains exactly one permutation avoiding 213 (its smallest element). The map sending a permutation to the maximal element of its L-class consists in sorting the Lcode of the permutation, whereas the map sending a permutation to the minimal element of its L-class consists in building, from right to left, the permutation of its Lcode that has the greatest possible value in each position. For example, with $\sigma=682547193$ whose Lcode is 561322010, the maximal element of its Lclass has Lcode 653221100, so is permutation 764352819, and the minimal element of its L-class has Lcode 016523210 , so is 139857642 .

\section{References}

[1] L. CARLitz, q-Bernoulli and Eulerian numbers, Trans. Amer. Math. Soc. 76 (1954), 332-350.

[2] A. CAYLEY, On the theory of the analytical forms called trees, Phil. Mag., (1857), 13:172-6.

[3] F. Chapoton, Algèbres pré-Lie et algèbres de Hopf liées à la renormalisation, C. R. Acad Sci. Paris, 332 Série I (2001), 681-684.

[4] A. Connes and H. Moscovici, Hopf algebras, cyclic cohomology and the transverse index theory, Commun. Math. Phys. 198 (1998), 198-246.

[5] H. FigueroA and J.-M. Gracia-Bondia Combinatorial Hopf algebras in quantum field theory I Rev. Math. Phys. 17 (2005) 881.

[6] D. FoATA and M. P. SChÜTZEnBERGER, Major index and inversion number of permutations, Math. Nachr. 83 (1970), $143-159$.

[7] I.M. Gelfand, D. Krob, A. Lascoux, B. Leclerc, V. S. RetakH, and J.-Y. Thibon, Noncommutative symmetric functions, Adv. in Math. 112 (1995), 218-348.

[8] F. HIVERT and N. THIÉRY, MuPAD-Combinat, an open-source package for research in algebraic combinatorics, Sém. Lothar. Combin. 51 (2004), 70p. (electronic).

[9] A. LASCOUX, Symmetric functions and combinatorial operators on polynomials, CBMS Regional Conference Series in Mathematics 99, American Math. Soc., Providence, RI, 2003; xii+268 pp.

[10] A. LAscoux, Schubert et Grothendieck: un bilan bidécennal, Sém. Loth. Comb. 50 (2004), B50i.

[11] M. LothaIRE, Algebraic combinatorics on words, Cambridge, 2002.

[12] P. A. MACMAHON, The indices of permutations and derivation therefore of functions of a single variable associated with the permutations of any assemblage of objects, Amer. J. Math. 35 (1913), 314-321.

[13] D.P. Rawlings, Generalized Worpitzky identities with application to permutation enumeration, Europ. J. Combin. 2 (1981), 67-78.

[14] M. SkAndera, An Eulerian Partner for Inversions, Sém. Lothar. Combin. 46 (2001) B46d.

[15] N.J.A. SLOANE, The On-Line Encyclopedia of Integer Sequences, http: //www.research. att.com/ njas/sequences/ 
